\title{
A Radial Basis Function for Registration of Local Features in Images
}

\author{
Asif Masood $^{1}$, Adil Masood Siddiqui ${ }^{2}$, and Muhammad Saleem ${ }^{2}$ \\ ${ }^{1}$ Department of Computer Science and Engineering, \\ University of Engineering and Technology, Lahore, Pakistan \\ ${ }^{2}$ Department of Electrical Engineering, University of Engineering and Technology, Lahore, \\ Pakistan \\ asif@uet.edu.pk, adiluet@gmail.com, drmsaleem@gmail.com
}

\begin{abstract}
Image registration based on landmarks and radial basis functions (e.g. thin plate splines) results in global changes and deformation spreads over the entire resampled image. This paper presents a radial basis function for registration of local changes. The proposed research was based on study/analysis of profile for different radial basis functions, supporting local changes. The proposed function was designed to overcome the weaknesses, observed in other radial basis functions. The results are analyzed/compared on the basis of different properties and parameters discussed in this paper. Experimental results show that the proposed function improves the registration accuracy.
\end{abstract}

Keywords: Radial basis function, Image registration, Compact support, Landmarks.

\section{Introduction}

Registration based on radial basis functions play an important role in medical applications, image warping and simulation of facial expressions. In this paper, we considered a point based non-rigid registration approach. Transformations based on radial basis function have proven to be a powerful tool in image registration. With this approach, the transformation is composed of radially symmetric function that serves as basis function. The choice of the radial basis function is crucial for overall characteristics such as the smoothness or the locality of transformation function.

An often applied non-rigid image registration is based on thin plate splines, introduced by Bookstein [1] for registration of medical images. Subsequently, Evans et al. [2] applied this scheme to 3D medical images and Goshtasby [3] applied it to 2D aerial image registration. This approach yields minimal bending energy properties measured over the whole image, but the deformation is not limited to regions where the point landmarks are placed. This behavior is advantageous for yielding an overall smooth deformation, but it is problematic when local deformations are desired. To cope with local deformations, the landmarks have to be well distributed over the images to prevent deformations in regions where no changes are desired [4]. 
The radial basis functions with compact support were designed to register the local deformations. These functions limit the influence of landmarks around a circular area. Computational efficiency is another advantage of such functions. A radial basis function with compact support was first introduced by Wendland [5]. It has been used to model facial expressions [6] and elastic registration of medical images [7]. Arad and Reisfeld [8] used Gaussian function to incorporate locality constraints by properly tuning the locality parameter. Some other functions are given in [9]-[13]. Disadvantage of these functions is that they do not properly span over the complete region of support. This may lead to deterioration of results, which are studied in this paper. The proposed radial basis function is designed to minimize these problems.

The criterion to evaluate the performance of radial basis functions is also proposed in this paper. On the basis of this evaluation criterion, results of proposed radial basis function are compared with similar functions i.e. Gaussian and Wendland. The proposed function proves better in all the results. Rest of the paper is organized as follow. Section 2 gives brief description of radial basis functions suitable for local deformation i.e. with compact support. The proposed radial basis function, its properties and analysis of results is discussed in section 3. Image registration results, with different radial basis functions, are presented/compared in section 4. Finally, section 5 concludes this presentation.

\section{Radial Basis Functions for Local Deformation}

Out of many approaches, Radial basis functions (RBF) is one mean of achieving scattered data interpolation, i.e. fitting a smooth surface through a scattered or nonuniform distribution of data points. RBF interpolation is a linear combination of radially symmetric basis functions, each centered on a particular control point. The value of the RBF is only a function of the distance from the center point, so that $\varphi(\mathbf{x}, \mathbf{c})=\varphi(\|\mathbf{x}-\mathbf{c}\|)$ or $\varphi(\mathbf{x})=\varphi(\|\mathbf{x}\|)$ if the function is centered at the origon. A function $\varphi$ that satisfies the property $\varphi(\mathbf{x})=\varphi(\|\mathbf{x}\|)$ can be categorized under radial basis functions, where the norm is Euclidian distance. For a given set of $\mathrm{N}$ corresponding radial basis functions has the following general form

$$
y(\mathbf{x})=\sum_{i=1}^{N} \omega_{i} \varphi(\|\mathbf{x}-\mathbf{c}\|)
$$

The radial basis function $y(\mathbf{x})$ is the linear combination of $\mathrm{N}$ radial basis functions, each having a different center $c_{i}$ and a weighing coefficient $\omega_{i}$. The word 'radial' reflects an important property of the function. Its value at each point depends on distance of the point from respective control point (landmark) and not on its particular position.

The radial basis functions can be broadly divided into two types, namely global and local. The global functions influence the image as a whole. This type of functions is useful when registration process needs repositioning and deformation of complete image. Some examples of global functions are thin plate splines $\left(\varphi_{T P S}\right)[1],[2]$, 
multiquadrics $\left(\varphi_{M Q}\right)$ [14], and inverse multiquadrics $\left(\varphi_{I M Q}\right)$ [15]. These functions are given in Table 1. These functions are global in nature and cannot be applied for local changes like simulation of facial expressions, or change in local features of body organs after a surgical operation. In these functions, location of each pixel is effected by each landmark, which is computationally time-consuming.

Table 1. Some Radial basis functions without compact support

\begin{tabular}{|c|c|c|}
\hline $\begin{array}{l}\text { Ser. } \\
\text { No. }\end{array}$ & $\begin{array}{l}\text { Radial Basis Function } \\
\text { without compact support }\end{array}$ & Profile \\
\hline 1. & $\begin{array}{l}\text { Thin Plate Splines (TPS) } \\
{[1],[2]}\end{array}$ & $\varphi_{T P S}(x)=x^{2} \log (x)$ \\
\hline 2. & Multiquadrics (MQ) [14] & $\varphi_{M Q}(x)=\sqrt{x^{2}+\sigma^{2}}$ \\
\hline 3. & $\begin{array}{l}\text { Inverse Multiquadrics (IMQ) } \\
\text { [15] }\end{array}$ & $\varphi_{I M Q}(x)=\frac{1}{\sqrt{x^{2}+\sigma^{2}}}$ \\
\hline
\end{tabular}

Radial basis functions with compact support can be very useful to deal with the local changes in an image. Influence of such functions is limited to a circular area around a landmark, which allows changes to a local area. A compactly supported radial basis function was established by Wendland [5]. It has been used to model facial expressions for video coding applications [6] and elastic registration of medical images [7]. Wendland's compactly supported function forms a family of radial basis functions that have a piecewise polynomial profile function and compact support. Member of the family to choose depends on the dimension $(d)$ from which the data is drawn and the desired amount of continuity $(k)$ of the polynomials. Fornefett et al. [7] shows with some proves that Wendland's radial basis function at $d=3$ and $k=2$ is most suitable for local deformation of images. He used it for elastic registration of medical images. This function is given as:

$$
\varphi_{F}(x)=(1-x)_{+}^{4}(4 x+1)
$$

Where $\varphi_{F}$ is the Wendland's function [5], used by Fornefett et al. [7]. They [7] compared the results with Gaussian function used by Arad and Deniel [8] to modify facial expressions in an image. The Gaussian function is given as:

$$
\varphi_{G}(x)=e^{-x^{2} / \sigma^{2}} \quad(\text { at } \sigma=0.5)
$$

The authors [7],[8] have demonstrated with examples that the two functions are very suitable for local deformation as these functions have the properties of primary interest in radial basis functions. These properties include locality, solvability, stability, and positive definite. We propose a new radial basis function that has compact support and can produce even better results. 


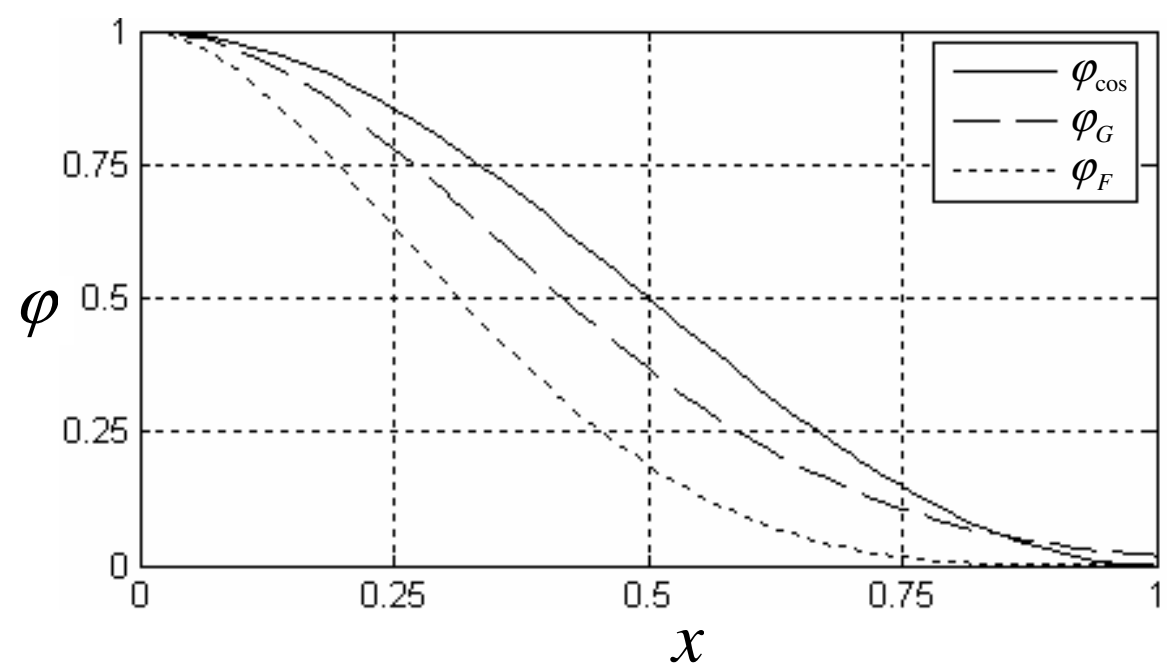

Fig. 1. Plot of three radial basis functions

\section{Proposed Radial Basis Function}

In local deformations, smooth transformation of image pixels is needed which should be proportional to the transformation of target landmark. A radial basis function maps the image pixels to their new location. The proposed radial basis function is based on study of its impact on smooth transition of pixels during local deformation. The proposed radial basis function is defined using cosine function. It is given as:

$$
\varphi_{\cos }(x)=\frac{1+\cos (x \pi)}{2}
$$

Plot of radial basis function $\left(\varphi_{\cos }\right)$ along with $\varphi_{F}$ and $\varphi_{G}$ is given in Fig. 1. Characteristics of radial basis function can be analyzed from the plot and their impact on individual points/pixels would be studied in later part of this section. Desired properties of radial basis functions and advantages of proposed function over others are discussed below (section 3.1).

\subsection{Properties of Radial Basis Function}

- Smooth transition from start to End: A radial basis function must be smooth at the start $(x=0)$ and end $(x=1)$. In other words, it must be along the horizontal line at its end points and should smoothly change its slope and join the two end points. It is important for smooth interpolation of effected image pixels. From Fig. 1, we can observe that all functions are smooth at its end points. The function $\varphi_{F}$ smoothes the end $(x=1)$ more than start $(x=0)$, which leads to deterioration of other properties discussed below. On the other hand, smoothness 
level remains same in proposed radial basis function. Although the function $\varphi_{G}$ is smooth along $x$ but it never reaches $\varphi=0$.

- Equal Distribution of $\varphi$ : Equal distribution of $\varphi$ is important to maintain the equal amount of impact on interpolating pixel points on both sides of target landmark. If this distribution is not controlled, some of the pixel points would map very close to each other and some would map far off from their neighbors. Proposed radial basis function produce ideal distribution of $\varphi$ along $x$. This is the inherent properties of cosine function which is used in this radial basis function. $\varphi=0.5$ at $x=0.5$ is one evidence of equal distribution. A straight line $(\varphi=1-x)$ can produce perfect distribution but it cannot be used as it would violate the first property discussed above. Distribution of both $\varphi_{G}$ and $\varphi_{F}$ concentrates more towards first half of $x$ i.e. $0-0.5$. About $63 \%$ of $\varphi_{G}$ falls at lower half of $x$. Similarly in case of $\varphi_{F}$, about $81 \%$ of points fall at lower half of $x$. This behavior would produce adverse effects on results, which is discussed in section $3.2 \& 3.3$. The proposed function $\varphi_{\text {cos }}$ distributes exactly $50 \%$ of points on both halves of $x$.

- Using full range of $x$ : The radial basis function should use the full range of $x$ i.e. $0-1$ for equal distribution of function $(\varphi)$. The proposed function $\left(\varphi_{\cos }\right)$ utilizes full range of $x$. The function $\varphi_{F}$ is almost 0 at $x=0.8$ and does not utilize the range properly from $x=0.8-1$. Similarly, $\varphi_{G}=0.18$ at $x=1$. In other words, it never produce $\varphi_{G}=0$ along the complete range of $x$ i.e. from $0-1$.

\subsection{Local Deformation on Single Row of Points}

This section demonstrates the application of radial basis function on single row of points. Impact of different radial basis functions, in the light of various properties presented in section 3.1, would also be studied. Deformation of (single row of) points after applying different radial basis functions $\left(\varphi_{\cos }, \varphi_{F}, \varphi_{G}\right)$ is shown in Fig. 2. The radial basis functions are applied to a row of 30 points using eq. 1 . The original location of points is shown in lowest row of Fig. 2. In this row of points, source $\left(\mathrm{P}_{1}\right)$ and target $\left(\mathrm{P}_{2}\right)$ location of landmark is inscribed in a circle and square respectively. As the deformation is based on single landmark, eq. 1 may be written as:

$$
y(\mathbf{x})=\omega^{*} \varphi(r)
$$

Where $\omega=P_{2}-P_{1}$, which is the displacement from source to target location. $\varphi(r)$ represents a radial basis function like $\varphi_{\cos }(r), \varphi_{G}(r)$, or $\varphi_{F}(r)$. In eq. $5, \mathrm{r}$ is the normalized distance from origin/center $(\mathrm{P} 1)$, which can range from 0 to 1 . It can be given as:

$$
r=\frac{\left\|\mathbf{x}-\mathbf{P}_{1}\right\|}{R}, \quad \text { and replace } \quad r=\{1: r>1\}
$$


From Fig. 2, $\mathbf{x}$ is the row of points that range from 1 to $30 . R$ is the radius around origin $\left(\mathrm{P}_{1}\right)$ that would be effected during deformation. It can be given as:

$$
R=\|\omega\| * a
$$

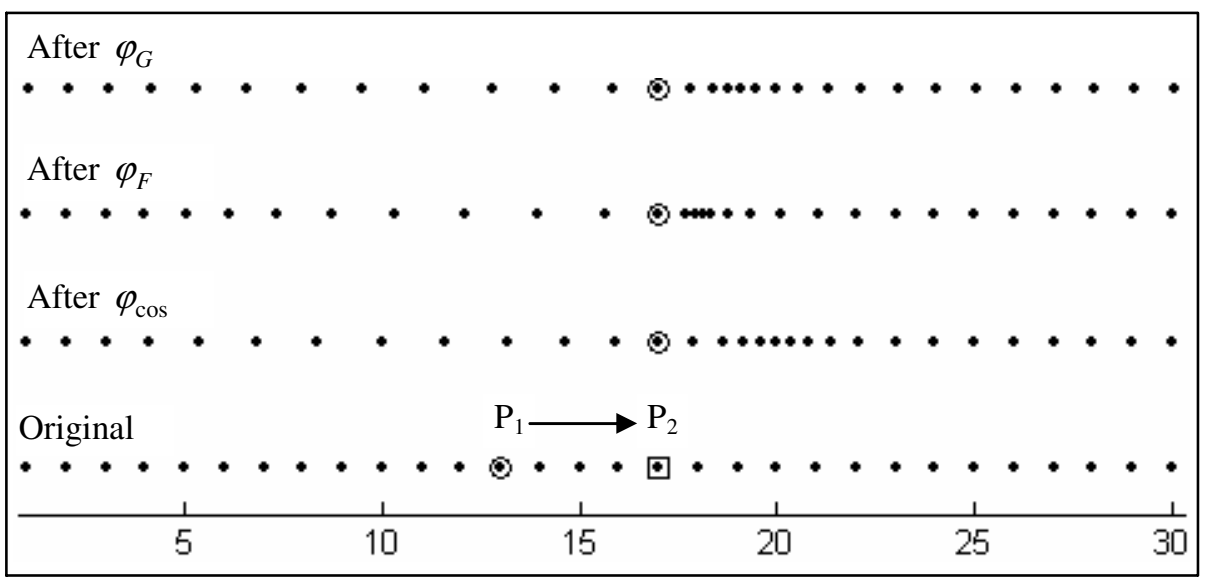

Fig. 2. Applying radial basis functions on a row of 30 points

The radius $\mathrm{R}$ is proportional to the displacement $(\omega)$ and ' $\mathrm{a}$ ' is the locality parameter. The parameter ' $\mathrm{a}$ ' is used to control the proportional extent of radius, which remains constant for all landmarks. After extensive testing, default value was set to 2.5. However, user may adjust the parameter 'a' as per its suitability in particular applications. Generally, parameter ' $a$ ' limits the locality of deformation and increasing the value of ' $a$ ' would tend to globalize the deformation effects.

Top three rows in Fig. 2 show local deformation after applying different radial basis functions. The point landmark (inscribed in circle i.e. $13^{\text {th }}$ point) moves from source $\left(\mathrm{P}_{1}\right)$ to target $\left(\mathrm{P}_{2}\right)$ location and all points with radius $R$ interpolate or adjust their location within available gaps. Proper utilization of available gaps and smooth change in distance between the points is desirable from a radial basis function. The properties of radial basis function (discussed in section 3.1) have direct impact on proper positioning of these points. We can compare the deformation results of different radial basis functions using parameters discussed below.

- Minimum distance (MinD): This is the minimum distance of any point from its neighbor. The radial basis function reduces the distance between points, if it needs to adjust them in smaller gaps. For example points at right side of origin in Fig. 2. This parameter is useful to monitor the adjustment in such situations. It can be given as:

$$
\operatorname{Min} D=\min _{i=1}^{n}\left\{d_{i}\right\}
$$

Where $d_{i}$, is the distance of $i$ th point from its neighbor. 
- Maximum distance (MaxD): This is the maximum distance of any point from its neighbor. This parameter is useful to monitor the adjustment of radial basis function when few points are to be settled in larger gaps. For example points at left side of origin in Fig. 2. It can be given as:

$$
\operatorname{Max} D=\max _{i=1}^{n}\left\{d_{i}\right\}
$$

- Maximum change in distance $(\operatorname{Max} \Delta D)$ : This shows the maximum change of distance between two points from their neighbors. This parameter monitors the smoothness of transition from larger gaps to smaller and vice versa. For example monitoring change in distance while moving from left side of origin to right side. It can be given as:

$$
\operatorname{Max} \Delta D=\max _{i=1}^{n}\left\{\Delta d_{i}\right\}
$$

Where $\Delta d_{i}$ is change in distance of $i$ th point.

A comparison of results for different radial basis function is shown in Table 2 . These results were calculated after deformation of row of 30 points shown in Fig. 2. The best value in Table 2 should have minimum difference from original i.e. before deformation. The best value for each parameter is written in bold. The first and third property of radial basis functions (i.e. smooth transition and using full range of $x$ ) has direct impact on parameter $\operatorname{Max} \Delta D$. We can see from Table 2 that $\operatorname{Max} \Delta D$ for $\varphi_{F}$ and $\varphi_{G}$ is about 3.3 and 1.6 times higher than $\varphi_{\cos }$. The second property (i.e. equal distribution) influences the parameter MinD and MaxD. From Fig. 1, we can observe

Table 2. Comparison of radial basis function applied on a row of points of Fig. 2

\begin{tabular}{l|c|c|c|c}
\hline $\begin{array}{l}\text { Evaluation } \\
\text { Parameters }\end{array}$ & $\begin{array}{c}\text { Before } \\
\text { Deformation }\end{array}$ & $\varphi_{\cos }$ & $\varphi_{F}$ & $\varphi_{G}$ \\
\hline MinD & 1 & $\mathbf{0 . 3 8 2}$ & 0.164 & 0.319 \\
\hline $\operatorname{MaxD}$ & 1 & $\mathbf{1 . 6 1 8}$ & 1.836 & 1.682 \\
\hline $\operatorname{Max} \Delta D$ & 0 & $\mathbf{0 . 1 9 6}$ & 0.652 & 0.314 \\
\hline
\end{tabular}

Table 3. Comparison of radial basis function applied on 2D grid of Fig. 3

\begin{tabular}{l|c|c|c|c}
\hline $\begin{array}{l}\text { Evaluation } \\
\text { Parameters }\end{array}$ & $\begin{array}{c}\text { Before } \\
\text { Deformation }\end{array}$ & $\varphi_{\cos }$ & $\varphi_{F}$ & $\varphi_{G}$ \\
\hline MinD & 1 & $\mathbf{0 . 5 5 7}$ & 0.405 & 0.517 \\
\hline $\operatorname{Max} D$ & 1.414 & $\mathbf{2 . 2 9 9}$ & 2.951 & 2.380 \\
\hline $\operatorname{Max} \Delta D$ & 0 & $\mathbf{0 . 2 1 5}$ & 0.222 & 0.238 \\
\hline
\end{tabular}


(a)

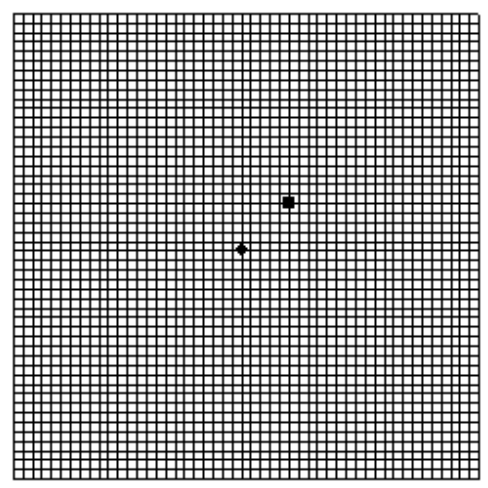

(c)

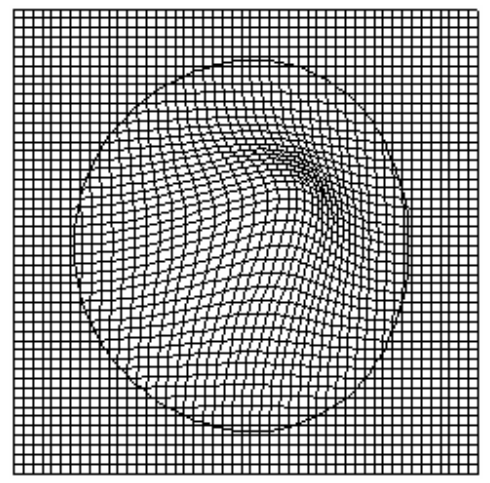

(b)

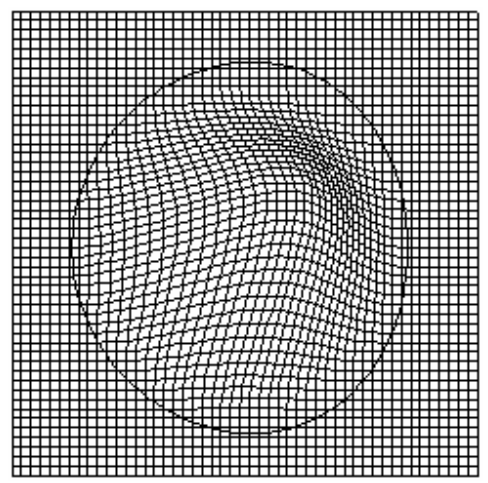

(d)

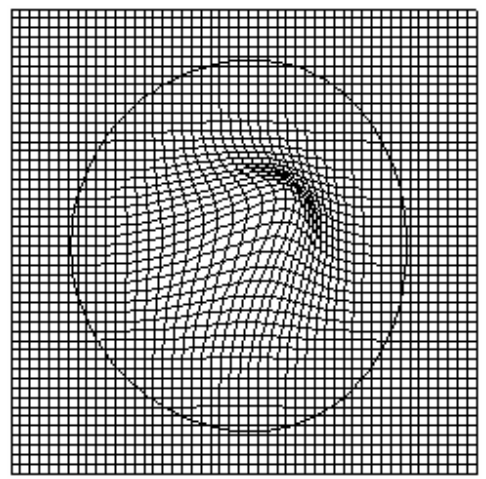

Fig. 3. Applying radial basis function on $50 \times 50$ grid. (a) Original grid marked with source (circle) and target (square) position of landmark, (b) After $\varphi_{\cos }$, (c) After $\varphi_{G}$, and (d) After $\varphi_{F}$.

that $\varphi_{\text {cos }}$ is best according to this property followed by $\varphi_{G}$ and $\varphi_{F}$. This can be verified from Table 2 also, as the MinD and $\operatorname{Max} D$ is best for $\varphi_{\cos }$ and worst for $\varphi_{F}$.

\subsection{Local Deformation on 2D Grid}

The application of radial basis function on single row of points is discussed in section 3.2. Same methodology can be extended to 2D grid of points. In $2 \mathrm{D}$ grid, each grid points (x) and landmarks (P1 and $\mathrm{P} 2)$ has the coordinates (x, y). The deformation of 50x50 grid with different radial basis functions $\left(\varphi_{\cos }, \varphi_{G}, \varphi_{F}\right)$ is shown in Fig. 3 . The radius $(\mathrm{R})$ around the center $(\mathrm{P} 1)$ is shown with a circle. One can observe that the grid after $\varphi_{\text {cos }}$ looks well distributed and smooth when compared with other two $\left(\varphi_{G}, \varphi_{F}\right)$. This can be verified by the quantitative measurements shown in Table 3 . 
Quantitative results are similar to the one shown in Table 2. The radial basis function $\varphi_{\text {cos }}$ again produces the best results which are shown in bold.

\section{Registration Results for Images}

Proposed radial basis function is designed to register local changes in an image. Such changes may include registration of post-operative medical images, simulation/ registration of tumor, kidney stones, and dislocation of bones. Some other

(c)

(a)
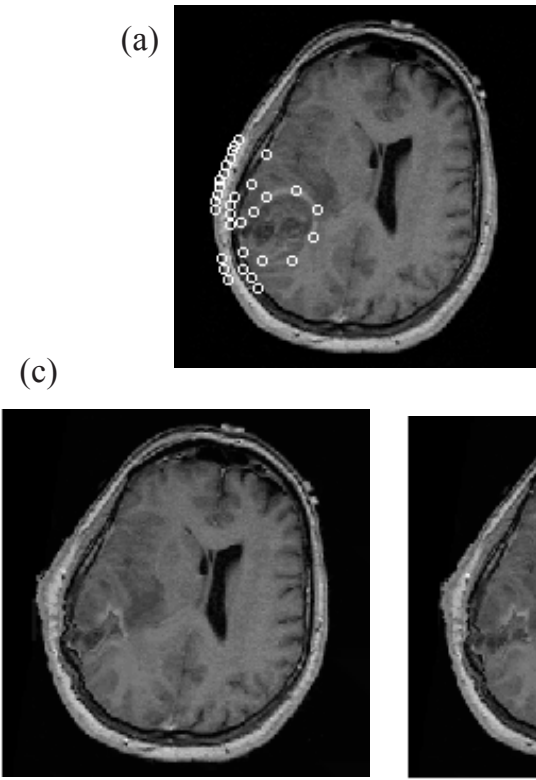

(f)

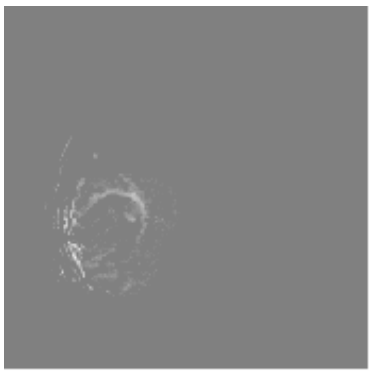

(d)

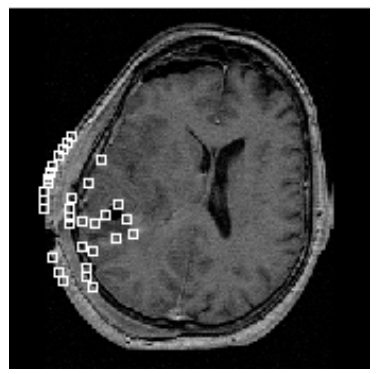

(b) (e)

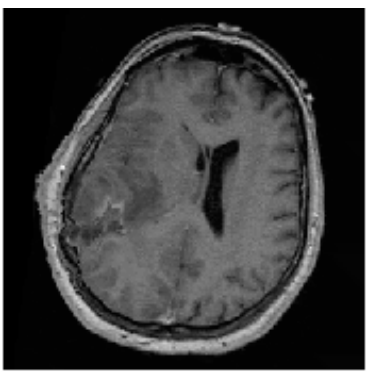

(g)

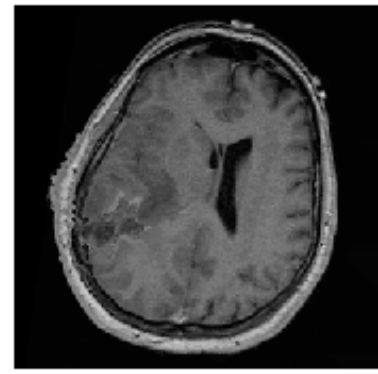

(h)
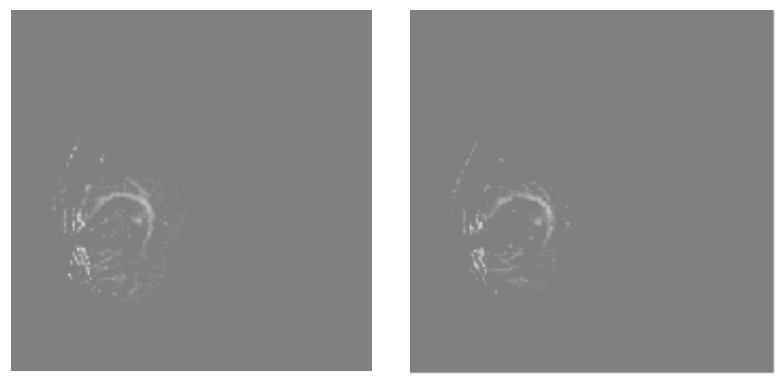

Fig. 4. Applying radial basis function on MR images. (a) Source image marked with source landmarks (circles), (b) Target image marked with target landmarks (squares), (c) Registered image with $\varphi_{\text {cos }}$, (d) Registered image with $\varphi_{G}$, (e) Registered image with $\varphi_{G}$, (f) Difference of (a) \& (c), (g) Difference of (a) \& (d), (g) Difference of (a) \& (e). 
applications may include image warping/morphing and simulation of facial expressions. As an example, registration of brain tumor is demonstrated in this section. Tomographic brain images, including pre-operative (Fig 4(a)) and postoperative (Fig. 4(b)) pictures, were taken from [7] for demonstration of results. The source (Fig. 4(a)) and target (Fig. 4(b)) images are corresponding slices of rigidly transformed 3D MR data sets. Aim of this registration is to correct the pre-operatively acquired image such that it agrees with the current anatomical situation. Registration results of proposed radial basis function $\left(\varphi_{\text {cos }}\right)$ are compared with $\varphi_{G}$, and $\varphi_{F}$. The source and target image with selected landmarks is shown in Fig. 4(a) and Fig. 4(b) respectively. The registered images with $\varphi_{\cos }, \varphi_{G}$, and $\varphi_{F}$ are shown Fig. 4(c), 4(d), and $4(\mathrm{e})$ respectively. Similarly, difference of registered image from original (Fig. 4(a)) is shown in Fig 4(f)-4(h). It shows that the radial basis functions ( $\varphi_{\text {cos }}$, $\varphi_{G}, \varphi_{F}$ ) obey the property of locality and effect only a limited area.

The results of these algorithms look very similar when compared with visual observation. Table 3 showed quantitative comparison of results for 2D grid. Measurement for these parameters (like $\operatorname{MinD}, \operatorname{MaxD}, \operatorname{Max} \Delta \mathrm{D}$ ) remain similar incase of images as well, since an image is also a 2D grid of pixels with different gray levels. Comparison of results in a graph is shown in Fig. 5. The parameters (in this graph) show the difference from original position (i.e. before deformation). This difference should be minimized. This graph shows that the results of proposed algorithm are best (i.e. having minimum value for each parameter), when compare with $\varphi_{G}$ and $\varphi_{F}$.

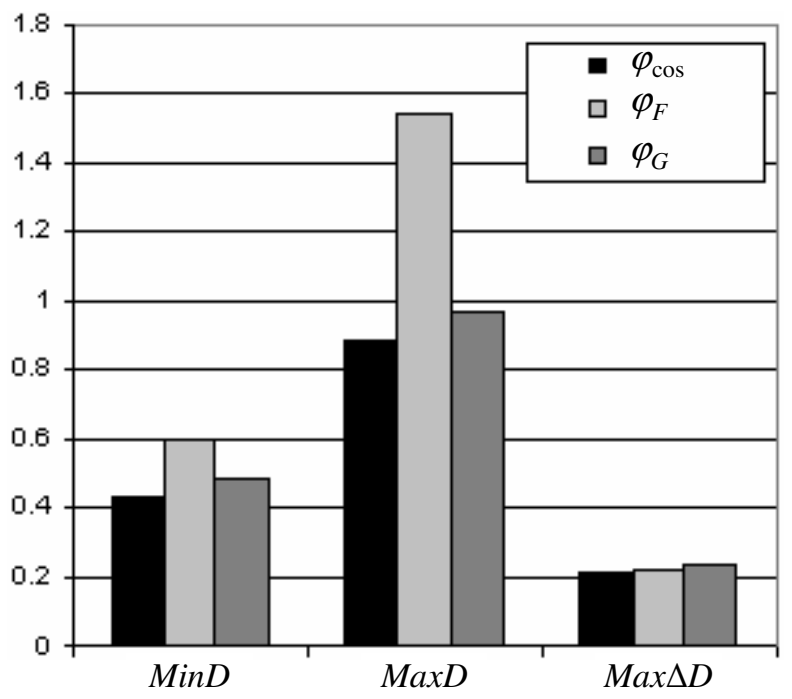

Fig. 5. Graph showing difference of parameters $\left(\varphi_{\cos }, \varphi_{G}, \varphi_{F}\right)$ from their original value (i.e. before deformation), for 50x50 grid 
(a)

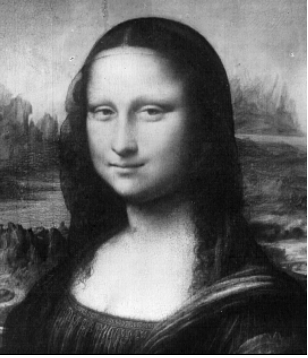

(c)

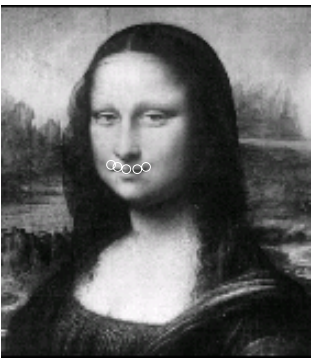

(e)

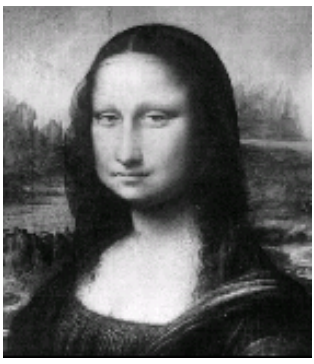

(h)

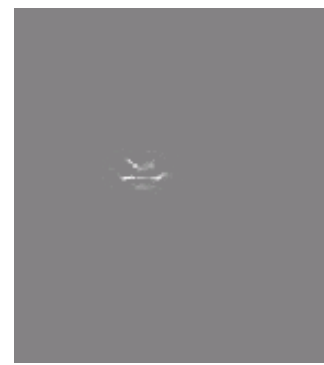

(f)

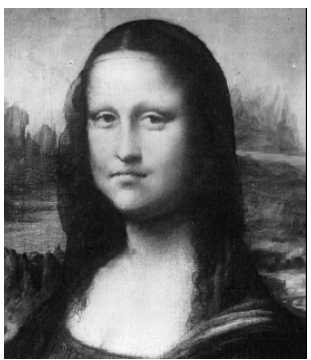

(b)

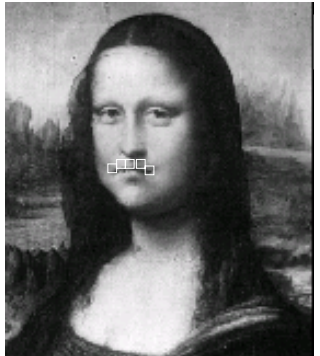

(d)

$(\mathrm{g})$

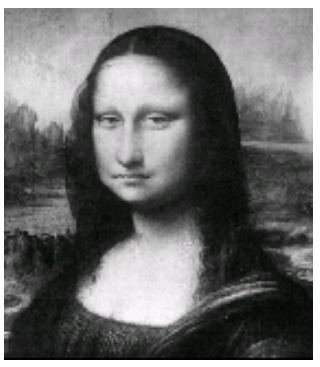

(i)

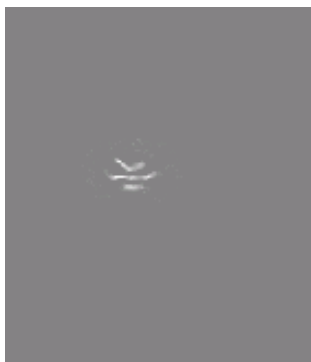

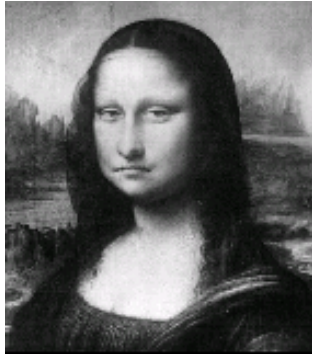

(j)

Fig. 6. Applying radial basis function on MR images. (a) Source image, (b) Target image , (c) Source image marked with source landmarks , (d) Target image marked with target landmarks , (e) Registered image with $\varphi_{\text {cos }}$, (f) Registered image with $\varphi_{G}$, (g) Registered image with $\varphi_{G}$, (h) Difference of a \& e, (g) Difference of a \& f, and (g) Difference of a \& g. 
Another set of result, i.e. registration of facial expressions, is shown is (Fig. 6). Fig. 6(a) is the famous Mona Lisa smiling image which is taken as source image. Fig. 6(b) shows the changed facial expression. This (Fig. 6(b)) was taken as target image. These images were taken from [8]. The marking of source and target landmarks is shown in Fig. 6(c) and 6(d). Registered images using different radial basis are shown from Fig. 6(e) - 6(g). Difference images are shown in Fig 6(h) - 6(j). Again the locality property of the radial basis functions is evident. Registration of facial expression produces the similar quantitative results shown in Table 3 and Fig. 5 . Thus, the values of quantitative measurements for the $\varphi_{\cos }$ are optimum followed by $\varphi_{G}$ and $\varphi_{F}$.

\section{Conclusion}

A radial basis function to register images with local deformations was presented in this paper. A study on desired properties of radial basis functions and different parameters to evaluate/compare the results was also presented. Deformation results were tested for row of points, 2D grid and images. Registered image is expected to improve similarity with target image. The proposed algorithm proved better in all the tests.

Acknowledgments. The authors acknowledge the Higher Education Commission (HEC) of Pakistan for providing funds for this research work and University of Engineering and Technology (UET), Lahore, Pakistan, for providing facilities to conduct this research.

\section{References}

1. Bookstein, F.L.: Principal warps: thin-plate splines and the decomposition of deformations. IEEE Trans. Pattern Anal. Mach. Intell 11(6), 567-585 (1989)

2. Evans, A.C., Dai, W., Collins, L., Neelin, P., Marrett, S.: Warping of a computerized 3-D atlas to match brain image volumes for quantitative neuroanatomical and functional analysis. In: Proc. SPIE, vol. 1445, pp. 236-246 (1991)

3. Goshtasby, A.: Ragistration of images with geometric distortions. IEEE Trans. Geosci. Remote Sens. 26(1), 60-64 (1988)

4. Goshtasby, A.: Image registration by local approximation methods. Image and Vision Computing 6, 255-261 (1988)

5. Wendland, H.: Piecewise polynomial, positive definite and compactly supported radial functions of minimal degree. Advances in Computational Mathematics 4, 389-396 (1995)

6. Soligon, O., Mehaute, A.L., Roux, C.: Facial expressions simulation with Radial Basis Functions. International Workshop on Synthetic-Natural Hybrid Coding and Three Dimensional Imaging, 233-236 (1997)

7. Fornefett, M., Rohr, K., Stiehl, H.S.: Radial basis function with compact support for elastic registration of medical images. Image and Vision Computing 19, 87-96 (2001)

8. Arad, N., Reisfled, D.: Image Warping using few anchor points and radial functions. Computer Graphics Forum 14(1), 35-46 (1995) 
9. Fornberg, B., Larsson, E., Wright, G.: A new class of oscillatory radial basis functions. Comput. Math. Appl. 51, 1209-1222 (2006)

10. Eickhoff, R., Ruckert, U.: Enhancing Fault Tolerance of Radial Basis Functions. IEEE Transactions on systems, Man and Cybernetics 35, 928-947 (2005)

11. Golberg, M.A., Chen, C.S., Bowman, H.: Some recent results and proposals for the use of radial basis functions in the BEM. Engineering Analysis with Boundary Elements 23, 285-296 (1999)

12. Šarler, B.: A radial basis function collocation approach in computational fluid dynamics. Computer Modeling in Engineering \& Sciences 7, 185-193 (2005)

13. Peng, W., Tong, R., Qian, G., Dong, J.: A Local Registration Approach of Medical Images with Niche Genetic Algorithm. In: 10th International Conference on Computer Supported Cooperative Work in Design, pp.1-6 (2006)

14. Little, J.A., Hill, D.L.G., Hawkes, D.J.: Deformations incorporating rigid structures. Computer Vision and Image Understanding 66(2), 223-232 (1997)

15. Ruprecht, D., Muller, H.: Free form deformation with scattered data interpolation method. Computing Supplementum 8, 261-281 (1993) 